\title{
La Formación Docente y la Educación de Jóvenes y Adultos: Análisis de la Práctica Pedagógica para la Enseñanza de Ciencias
}

\author{
Lorenna S. O. Costa(1), Victor F. A. Barros ${ }^{(2)}$, Márcia C. R. Lopes ${ }^{(1)}$ y Luciana P. Marques(1) \\ (1) Instituto Federal de Educação, Ciência e Tecnologia de Goiás (IFG), Câmpus Inhumas, Brasil. \\ (e-mail: Iorenna.silva@ifg.edu.br; luciana.marques@ifg.edu.br; marcia.lopes@ifg.edu.br)) \\ (2) Escola de Engenharia, Universidade do Minho, Azurém, Guimarães-Portugal. \\ (e-mail: vfbarros@dsi.uminho.pt)
}

Recibido Jun. 30, 2014; Aceptado Ago. 22, 2014; Versión final recibida Dic. 1, 2014

\begin{abstract}
Resumen
Se discute y analiza el proceso de elaboración e implementación de un proyecto que propone la enseñanza interdisciplinar de la ciencia considerando discusiones sobre problemas sociales en un curso del Programa Nacional para la Integración de la Educación Profesional con la Educación Básica en la Modalidad de Educaión de Jóvenes y Adultos, PROEJA, en el área de Informática del Instituto Federal de Educación, Ciencia y Tecnología de Goiás en Brasil. Se detallan las tres primeras etapas del estudio, enfocando la elaboración y aplicación de una propuesta didáctico-pedagógica para la enseñanza de ciencias, por profesores formadores y profesores en formación inicial y continuada, en que la interacción entre los participantes posibilitó la integración entre teoría y práctica. En la realización de este proyecto se demostró que los contenidos pueden ser trabajados en una forma contextualizada e interdisciplinar para que las contribuciones curriculares puedan reducir la dualidad entre formación general y formación técnica existente en la educación tecnológica.
\end{abstract}

Palabras clave: educación científica, educación ambiental, educación de jóvenes y adultos, formación de profesores

\section{Teachers Formation and the Education of Young and Adult People. Analysis of a the Pedagogical Practice for the Teaching of Science}

\begin{abstract}
The design and implementation of a project aimed to improve the teaching of science incorporating discussions on social problems in a course belonging to The National Educational Program for Young and Adult People, PROEJA. The subject of the course is informatics of the Federal Institute of Education Science and Technology of Goiás in Brazil (IFG). The first steps of the study are described focusing on the elaboration and application of the pedagogical-didactic proposal to teach sciences by new teachers in which the interaction between the participants allowed the integration between theory and practice. During the development of this project it was shown that the contents can have a contextual and interdisciplinary approach so curricular contributions can reduce the duality of general formation and technical formation currently in used in technological education.
\end{abstract}




\section{INTRODUCCIÓN}

Sin restar importancia al tratamiento sistemático de la pluralidad cultural y de conocimientos en el currículo, somos defensores de la idea de que la educación científica tiene un papel destacado en la configuración social, política, económica y ambiental contemporánea. Para que el estudio de las ciencias contribuya a una formación integral y de carácter transformador para los alumnos, debemos ir más allá de la transmisión del conocimiento científico habitual. Así pues, se debe incluir un acercamiento a la naturaleza de la ciencia y la práctica científica vinculando ciencia, tecnología, sociedad y medio ambiente, con el fin de fomentar la participación fundamentada de los ciudadanos en decisiones razonadas (Cachapuz, Gil-Perez, Carvalho y Vilches, 2005). Por lo tanto, la educación científica puede proporcionar aportes significativos a la formación de ciudadanos críticos, capaces de desvelar y enfrentarse a las contradicciones de la sociedad (Mortimer, Machado y Romanelli, 2010).

En Brasil, sin embargo, percibimos un gran problema relacionado con la realidad educativa, que hace aún más necesario reflexionar y valorar el trabajo en la educación científica en las escuelas. Los datos indican que aproximadamente catorce millones de personas con quince años o más son analfabetos. Si, además nos centramos en aquellos que han tenido hasta cuatro años de estudio en ese rango de edad (analfabetos funcionales) suman cerca de treinta y tres millones de habitantes (IBGE, 2010). Frente a esta realidad, brevemente esbozada, el gobierno brasileño ha promovido acciones para intentar reducir tal problemática. Con este propósito en 2005 fue creado un programa dirigido a la población joven y adulta que tenía como fin promover la formación básica vinculada a la formación profesional, el Programa Nacional para la Integración de la Educación Profesional con la Educación Básica en la Modalidad de Educación de Jóvenes y Adultos (PROEJA). EI PROEJA pretende rescatar y promover la reinserción de millones de jóvenes y adultos en el sistema educativo brasileño, facilitándoles el acceso a la educación y la formación profesional en la perspectiva de formación integral. De esta forma, ciertos fundamentos político-pedagógico conllevan la organización curricular para el cumplimiento de esta política pública, como por ejemplo, la integración curricular con miras a la calificación profesional y social, orientada al incremento de la tasa de escolaridad (BRASIL, 2006).

También se destaca la escasez de docentes capacitados en el área de Ciencias en Brasil, debido a la gran devaluación de este trabajador. Esta profesión es cada vez menos adoptada por los jóvenes brasileños. A partir de 2008, a través de la expansión de la Red Federal de Educación Profesional y Tecnológica, fueron creados los Institutos Federales de Educación, Ciencia y Tecnología, se produjo el crecimiento de la oferta de plazas en cursos de capacitación para profesores, ya que estas instituciones deben destinar como mínimo un $20 \%$ de sus cupos para estos cursos. Sin embargo, observamos que, precisamente en los cursos de formación de profesores de química y física, áreas en las cuales ya hay pocos profesionales disponibles, destaca la poca demanda y una pronunciada evasión (Lima, 2013).

Con el objetivo de mejorar la formación de profesores y valorarlos, en 2007 fue creado el Programa Institucional de Becas de Iniciación a la Docencia (PIBID). EI PIBID prevé la asociación entre dos instituciones: una de grado superior, en la que el profesor coordinador y los alumnos de graduación están localizados, y una escuela de enseñanza básica, que será el campo elegido para la implementación del programa. El profesor coordinador dirige a los alumnos de la graduación a actuar en el seno de escuela bajo la inspección de un profesor supervisor de esta unidad, experimentando, como futuros educadores, la compleja realidad. Estas acciones son ejecutadas incluso antes de las actividades relacionadas con el curso obligatorio de prácticas didácticas.Tanto el PIBID como el PROEJA se están desarrollando en diversas instituciones de educación superior en el país. El Campus Inhumas del Instituto Federal de Educación, Ciencia y Tecnología de Goiás (IFG) ejecuta el programa PIBID desde 2009 y actualmente tiene 14 becarios. También ofrece un curso PROEJA de Soporte Técnico y Mantenimiento de Equipos, que tiene como uno de sus varios objetivos, realizar el mantenimiento preventivo y correctivo de equipos de computación, identificando los principales componentes de una computadora y sus funcionalidades.

Siendo la educación un campo de disputas y la escuela, de acuerdo con Gómez (2002), muchas veces, un aparato de hegemonía, creemos que a través de la misma es posible tanto inculcar como cuestionar supuestos de la ideología dominante. Se trata pues, de proponer otra racionalidad a los desafíos contemporáneos, buscando una práctica educacional basada en los presupuestos de desarrollo social y humano que contribuyan a la preservación ambiental y la equidad. Es en esta última perspectiva donde hemos trabajado.

Algunos estudios revelan que los principales problemas en la educación de jóvenes y adultos son la falta de adecuación del currículo y la ausencia de una propuesta de intervención pedagógica que satisfaga las necesidades de este público (Costa, 2010). Por otro lado, no hay muchos profesionales dispuestos a pensar en un proyecto que tenga en cuenta las especificidades de esta modalidad educacional. El efectivo proceso 
de evaluación de la enseñanza presupone el tratamiento de problemas pedagógicos con claridad, coherencia e interés por la aprehensión de la realidad vivida por los estudiantes a partir de la consciencia de las contradicciones presentes en la sociedad en que viven, sólo así se puede construir un trabajo que mejore el proceso de enseñanza-aprendizaje (Barros, Orilla, Castro y Hijo, 2013).

Todo ello indica que lo que ocurre en Brasil, hasta el momento, es que la mayoría de las disciplinas, metodologías y estrategias usadas en las aulas no son adecuadas para las especificidades de los alumnos jóvenes e adultos. En este contexto, desde el enfoque de la formación realizada en el PROEJA y con el objetivo de contribuir a la formación de docentes en el IFG, se propuso la elaboración e implementación de un proyecto didáctico-pedagógico con estudiantes y profesores implicados en el PIBID. Para ello se partió de la propuesta "Desecho electrónico: retos en la sociedad de consumo", que propone la enseñanza interdisciplinar de ciencia y el abordaje de discusiones sobre problemas sociales en el curso PROEJA ya mencionado. Este artículo pretende discutir y analizar el proceso de elaboración y algunos aspectos de la implementación del referido proyecto, presentando estrategias usadas en las clases del PROEJA y su evaluación.

\section{METODOLOGÍA}

Esta investigación, de tipo cualitativo, fue desarrollada en el ámbito del Núcleo de Estudios y Pesquisas en Enseño de Ciencias (NEPEC) del IFG - Campus Inhumas y metodológicamente caracterizada como una investigación-acción (Tripp, 2005). El intento de señalar la dirección de una propuesta didáctico-pedagógica diferenciada que venga a crear un diálogo entre el contenido a trabajar y las experiencias de los estudiantes se basa en la pedagogía de Freire (2005). Utilizamos también los presupuestos teóricos de Vygotsky (2001) porque compartimos con este teórico la idea de que la formación de los conceptos científicos se originan desde los conceptos espontáneos.

Con el objetivo de desarrollar y orientar las actividades de investigación, se realizaron ocho reuniones quincenales en el segundo semestre de 2012, todas ellas registradas en audio y video y, posteriormente, transcritas para análisis. Participaron de las reuniones profesores formadores - PF, profesores en formación inicial - PFI (estudiantes ligados al PIBID) y profesores en formación continua - PFC (profesores del PROEJA de las disciplinas de Química, Biología, Matemáticas, Informática e Historia). La investigación se llevó a cabo en cinco pasos: 1) percepción y diagnóstico de una situación de enseñanza problemática que se pretendía mejorar o resolver; 2) formulación de estrategias de acción; 3) desarrollo de esas estrategias y evaluación de su efectividad; 4) ampliación de la comprensión de la nueva situación; 5) repetición de mismos pasos para la nueva situación práctica (Pereira, 1998).

Los pasos 1 y 2 fueron ejecutados por medio de reuniones periódicas, con la finalidad de analizar el curso PROEJA y planear el proyecto didáctico-pedagógico, buscando pensar y discutir si habría una necesidad real de definir un abordaje diferenciado de la práctica pedagógica para el PROEJA. El paso 3 tenía como propósito el desarrollo de este proyecto que se considera un tema de la experiencia de los estudiantes del curso PROEJA y su análisis. Presentaremos los pasos 1, 2 y 3 . Por su parte, los pasos 4 y 5 no serán descritos en este trabajo.

\section{RESULTADOS Y DISCUSIÓN}

\section{La elaboración de la propuesta: interacción dialógica en la construcción de significados}

Analizando las transcripciones, se pudo identificar discursos recurrentes en las enunciaciones que permitió una construcción de categorías y conceptos que los PF, PFI y PFC trajeron de su experiencia, profesional o académica, con el PROEJA. Estas categorías fueron fundamentales para la elaboración del proyecto didáctico-pedagógico, porque posibilitaron identificar sus visiones de educación así como sus necesidades de formación para el trabajo en la educación de jóvenes y adultos (EJA). Se estructuraron tres categorías: 1) Tipos de Discurso; 2) Enfoques del Discurso sobre la caracterización del PROEJA; y 3) Dimensión de la Práctica Docente. Por su importancia en las decisiones en el proceso de construcción del proyecto, la discusión de la categoría 3 será el foco de este trabajo (tabla 1).

Autores reconocidos en el área de formación docente (Schön, 1997; Tardif, 2002) dan énfasis a la epistemología de la práctica como un modelo de formación del profesorado. Sin embargo, basados en autores de la teoría histórico-cultural, como Vygotsky (2001) y Duarte (2003), se puede afirmar que la apreciación de los conceptos espontáneos, producidos en la práctica educativa, debe ser sólo el primer paso de la formación inicial y continua de docentes. Conocimientos teóricos, que agreguen conceptos científicos son importantes en la constitución de estos profesionales. En esta dirección las discusiones en grupo fueron orientadas por escritos del área de educación de jóvenes y adultos con enfoque en la 
enseñanza de la ciencia así como otros relacionándose al tratamiento de residuos electrónicos. Hubo un esfuerzo deliberado para considerar los conocimientos específicos de cada área, en una interacción multidisciplinar, contribuyendo a la expansión del análisis de la complejidad del trabajo docente.

Tabla 1: Dimensión de la Práctica Docente

\begin{tabular}{|l|l|}
\hline Dimensión de la Práctica Docente & Definición \\
\hline Presupuestos de la Práctica & $\begin{array}{l}\text { Presupuestos teóricos o no, que guían la planificación y la } \\
\text { acción del profesor. }\end{array}$ \\
\hline Situación Didáctica & $\begin{array}{l}\text { Acción docente en el aula teniendo en cuenta la } \\
\text { interacción profesor-alumno. }\end{array}$ \\
\hline Reflexión Docente & Reflexión del profesor sobre su práctica. \\
\hline
\end{tabular}

La Reflexión sobre la enseñanza, que hizo hincapié en los problemas que los profesores enfrentan en el aula del PROEJA, ofreció un elemento de destaque en las reuniones (fragmento 1).

\section{Fragmento 1}

3. PFC3: La primera vez que trabajé fue en el primer semestre de 2010. Después, no volví más a trabajar con el PROEJA por un par de semestres He vuelto a trabajar y estoy ya hace un tiempo trabajando con ellos. Me he dado cuenta que tenemos que conocer un poco más, aprender un poco más sobre ellos, comprender la situación de estos estudiantes y intentar desarrollar herramientas, desarrollar metodologías que sean más, ¿cómo diría?, más apropiadas para estos alumnos. [...] Porque no hemos alcanzado nuestros objetivos, la evasión y repetición, de cierta manera, demuestran esto...

Analizando el discurso de PFC3, la afirmación de que "ha entendido" la necesidad de saber más sobre los alumnos de la EJA, indica que esta conclusión no se construyó al instante y ni por estudios teóricos sobre el tema. Al contrario, se originó a partir del desarrollo de los conceptos espontáneos, vinculada a su acción en el aula. Sin embargo, habiendo decidido este profesor participar en las reuniones, se puede pensar que hubo la constatación de una necesidad de conocimientos científicos para apoyar su acción. Así, si el contenido de una formación "se reduce fuertemente a una reflexión sobre el conocimiento profesional, de carácter tácito, personal [...]" (Duarte, 2003, p. 620), las reuniones producirían poco resultado. Los conocimientos necesarios a la práctica docente están más allá de la experiencia. Así, la reanudación de los estudios, a través de educación continua, ofrece una rica interacción entre las necesidades percibidas en el aula y los conocimientos teóricos de la academia.

Aún en el turno 3, PFC3 se plantea problemas relacionados a la EJA: alusión y desaprobación. Las análisis históricas nos revelan que la escuela no reconoce las especificidades de estos estudiantes, que un día fueron excluidos del derecho a la educación (Oliveira, 2013). Al contrario, a menudo, se refuerza y se reproduce esta exclusión. Una de las causas de este problema, por ejemplo, son propuestas curriculares que permiten la fragmentación del conocimiento, orientación que debe ser reconsiderada en los cursos de formación.

En esto mismo turno, PFC3 se refiere también al desarrollo de herramientas y metodologías más apropiadas para los alumnos. La frecuente pregunta ¿Cómo se hace...? entre los profesores señaló la necesidad de tener materiales didácticos más específicos para trabajar con el PROEJA. En este sentido, una dimensión reiteradamente enunciada por los profesores es la Situación didáctica, que implica la acción del docente en el aula. Tanto los PFI cuántos los PFC, hablan de la necesidad de tener métodos específicos para el trabajo, destacando la perspectiva de "cómo" tratar la enseñanza en el PROEJA (fragmento 2).

\section{Fragmento 2}

2. PFC1: [...] trabajaba en un proyecto anterior con 'basura electrónica', después de algunas visitas técnicas percibimos la necesidad de saber un poco más acerca de esta parte, involucrando química y problemas con la enseñanza en el PROEJA. Porque la visita era muy "técnica". Entonces, ¿cómo trabajar?

4. PFI1: [...] la primera vez que estuvimos allí (refiriéndose a la clase de química en el período de pasantía) es muy diferente a lo que hemos visto en la escuela secundaria y en la educación superior. A veces la explicación es rápida y hay que explicar nuevamente, pues no entienden. ¿Cómo hacer? Es difícil... 
Entendemos la necesidad de discutir las metodologías para su utilización en la clase que, con el cuidado de que no incurran en un modelo tecnicista, inspiren debates curriculares conectados a una perspectiva crítica del currículo. La dimensión del "cómo" -prioridad de las perspectivas técnicas - no estará ausente, pero será concebida en sus múltiples relaciones con otros temas, destacados y centrales en la teorización crítica, a saber: Qué, por qué, para quién y quién decide.

En este sentido, PF1 hace una interrogación sobre la elección de los contenidos de las disciplinas y PFC3 contribuye con un ejemplo (fragmento 3).

\section{Fragmento 3}

61. PF1: el tiempo de carga de química es reducido [...] y luego se intenta condensar el contenido de un año en seis meses, no se repiensa la práctica o se replantean contenidos que se abordarán, ni como serán abordados [...]

75. PFC3: [...] la disciplina de matemáticas es de alguna manera privilegiada en el curso PROEJA, porque es la que tiene mayor carga horaria [...] y no es posible verlo todo. Yo, por semestre, no puedo trabajar más de la mitad del contenido.

78. PFC2: Creo que es necesario revisarlo, porque en química, incluso en la secundaria, es demasiado contenido. No se necesitaría todo [...]

79. PFC3: No creo que tengamos que preocuparnos por terminar todo el contenido, el alumno tiene que aprender... [...]

81. PFC3: Tal como es en el proyecto (refiriéndose al proyecto del curso PROEJA), también me veo así... preocupado por no estar cumpliendo con lo que está escrito... porque el proyecto es aquel y, de alguna manera, al estudiante se le está engañando.

Se observa en este fragmento, un discurso "por decir" acerca de los supuestos de la práctica. El conflicto de PFC3 contempla el reconocimiento de la imposibilidad de concluir todo el contenido propuesto en el diseño del curso, la preocupación por no estar "cumpliendo lo que está escrito" y engañar a los estudiantes. PFC3 manifiesta su intranquilidad y preocupación con el aprendizaje, demostrando una visión crítica de la educación. Tal vez sus conclusiones se hayan originado a partir de su propia vivencia escolar, juntamente con la reflexión que llevó a cabo durante los años de trabajo o a través de lecturas del área educativa de línea crítica. Sin embargo, en el turno 81, PFC3 retrocede y demuestra la inseguridad por no lograr el cumplimiento de los contenidos. Se manifiesta la necesidad de estudios teóricos que basen su argumentación y justificación en la no priorización del contenido sobrante y sí en el aprendizaje.

Cuando se piensa en proyectos didáctico-pedagógico basados en los temas de la experiencia, se necesita revisar qué contenidos son realmente necesarios, cuál debe el grado de complejidad adecuado y, con miras a un trabajo interdisciplinar, establecer las relaciones multidisciplinares. Así, el profesor necesita autonomía para decidir el contenido que va a trabajar, pero esa decisión no debe ser individual y si el producto de acuerdos pactados en reuniones colectivas con grupos de profesores. El profesor de historia o de matemáticas, ciencias o estudios sociales [...] contribuyó específicamente, a la luz de la democratización de la sociedad brasileña, atendiendo a los intereses de las clases populares, a la transformación estructural de la sociedad. [...] dicha contribución será más eficaz si el profesor es capaz de entender los lazos de su práctica con la práctica social global. (Saviani, 2008, p. 64).

Así, la preocupación central del profesor debe ser el aprendizaje de conceptos científicos relacionados con temas de la experiencia. "Es necesario no tener miedo de innovar. Ante el fracaso generalizado de la enseñanza de las ciencias, deben elaborarse e implementarse nuevas propuestas, pero las innovaciones deben ser cuidadosamente elaboradas" (Echeverría; Santos; Costa, 2012). Fue posible identificar que varias acciones de los docentes en el aula y las ideas de los profesores en formación se basaban en criterios subjetivos y a menudo no lograron explicar o justificar sus acciones (fragmento 4).

\section{Fragmento 4}

205. PFC1: Yo trato de hacer lo que quería que hubieran hecho conmigo...

Sin embargo, para la acción docente es esencial la toma de consciencia. Vygotsky (2001), para discutir el desarrollo de las funciones psicológicas superiores, afirma que el sujeto domina estas funciones al mismo tiempo que se intelectualiza, y la toma de conciencia es parte de ese proceso. 
A partir de estas reflexiones, sobre la necesidad de reducir el contenido del pensamiento en el aprendizaje, considerando los tiempos específicos de los alumnos del PROEJA así como los temas de su vivencia, se preparó el proyecto "Basura Electrónica: reto en la sociedad de consumo", que tuvo como tema transversal la educación ambiental, como podemos ver en el fragmento 5.

\section{Fragmento 5}

206. PF1: Pensemos juntos cómo hacer y cómo funciona...

208. PFC1: Entonces, he trabajado con el proyecto de basura electrónica, pero, como he dicho, desde otra área, química, por ejemplo...

206. PFC3: En matemáticas podemos analizar la cantidad de basura producida... puedo cambiar mi contenido, mi planificación. Necesito tiempo.

207. PF1: Bueno... supongo que es posible, entonces tenemos que pensar en los objetivos del proyecto, el tiempo, como trabajar... Algunas cosas no sé sobre el tema de la basura... Algo de lo que tenemos que hablar es sobre la explotación del trabajo. Esta es una discusión de todos, ¿cierto?

208. PF2: También creo, pero podríamos pensar en acciones integradas, las visitas técnicas...

209. PFC1: lo hemos hecho, en el centro de re-acondicionamiento, fue muy bueno. Podemos pensar.

A lo largo de las ocho reuniones se ha estructurado el proyecto didáctico-pedagógico, definiendo los objetivos, los conceptos a estudiar, que serán discutidos a seguir.

\section{El proyecto didáctico-pedagógico: un informe de la experiencia}

Partiendo de las discusiones anteriores y utilizando el aporte teórico elaborado por Costa et al. (2012) se destacaron algunas de las condiciones diferentes de estos individuos, tales como: tiempo específico de aprendizaje; dificultad en el trato a nivel del pensamiento abstracto; falta de una cultura escolar de estudio; alta experiencia y riqueza del conocimiento cotidiano; entre otros. Estos aspectos fueron decisivos para la elaboración del proyecto.

El proyecto fue desarrollado en ocho clases de 1,5 horas, en el primer período del curso PROEJA. Textos y películas como, por ejemplo "La historia de las cosas", fueron abordados con los alumnos con el fin de relacionar los estilos de vida de los estudiantes con las cuestiones ideológicas en nuestra sociedad. La necesidad de repensar el consumo fue otro asunto trabajado, evitando caer en un "ecologismo ingenuo", en el cual a cada uno se le pide que haga su parte, haciendo caso omiso de que la parte que le toca a cada uno en la injusticia ambiental es diferente (Porto-Gonçalves, 2011). De esta forma, se consideró el consumo de cada país, así como las desigualdades en el interior de nuestra comunidad. De esta manera, queda claro que la producción de residuos y su posterior depósito en el medio ambiente es actualmente un aspecto bastante propicio para discutir en el escenario educativo propuesto.

Varios conceptos podrían ser elegidos, como las propiedades generales y específicas de cada material, la historia de los metales en el desarrollo de la humanidad, sustancias, problemas causados por su eliminación incorrecta, metales y organismos vivos, preciosidad de los metales, diferencia entre el vertederos: rellenos sanitarios y vertederos controlados, entre otros. También se trabajó con el problema del tratamiento y eliminación de residuos electrónicos, especialmente en países donde hay una inversión masiva en tecnología para la gestión de los residuos. El grupo ha analizado esas discusiones en la realidad de la ciudad de Inhumas, Goiás (Brasil), contextualizando un debate social y ambiental en el que emergieron algunas preguntas, como: ¿Por qué no invertir en tratamiento de aguas residuales? ¿Qué problemas ambientales se derivan de la ausencia de tratamiento? ¿Qué problemas de salud puede tener o ya tiene la población? La Figura 1 presenta la estructura general do proyecto.

En el proyecto se señalizó la importancia de repensar la Educación Científica teniendo en cuenta la complejidad de la Educación Ambiental, así como qué implicaciones tienen la producción y la eliminación de los desechos electrónicos. Por lo tanto, ante estas discusiones, se propuso que en el tratamiento de las disciplinas del área básica, desde el primer semestre del curso, se discutieran temas relacionados con la experiencia de los estudiantes y el área de informática. De esta manera, buscamos valorar las preocupaciones alrededor de los espacios del trabajo y de la vida en sociedad. A continuación se plantearon lecciones teniendo en cuenta dos cuestiones principales: la producción y el descarte de los desechos electrónicos y la importancia de la Educación Científica para jóvenes y adultos. 


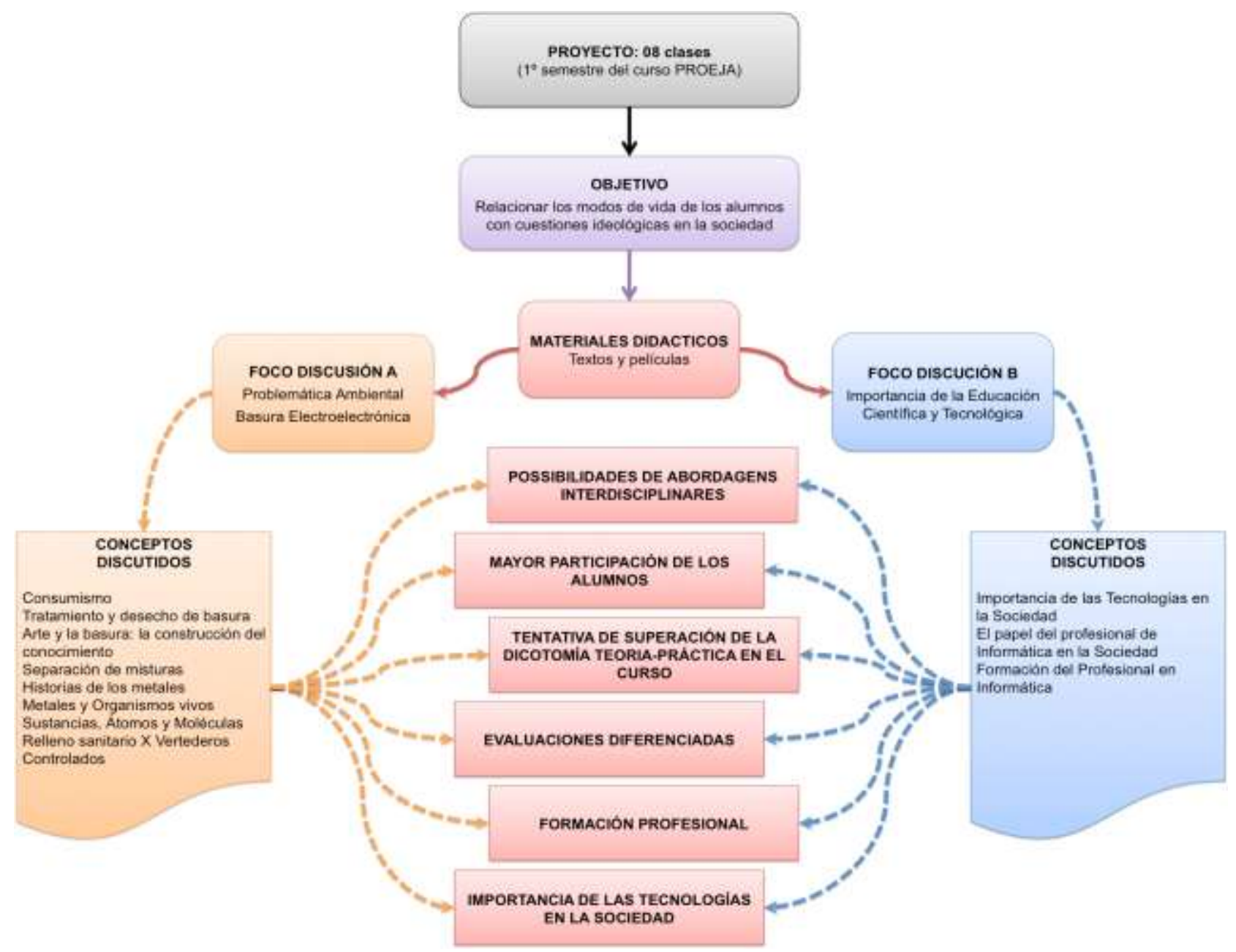

Fig. 2: Guión General y Resultado de la Investigación.

Con relación a la Producción y Eliminación de los Desechos Electrónicos se discutieron con los estudiantes los siguientes temas: hacia adónde irían estos materiales después de su descarte, los resultados que provocaría su contacto con el suelo si descartados de forma incorrecta etc. Estas discusiones se hicieron con los profesores de modo interdisciplinar. A los estudiantes se les pidió que trajeran equipos electrónicos (AEE) que ya no tuvieran uso. De esta forma, computadoras fueron desmontadas para que los alumnos estudiaran la placa base y los materiales que la constituyen. Se trabajaron varios conceptos como propiedades generales y específicas de cada material; historia de los metales en el desarrollo de la humanidad; sustancias; problemas causados por una eliminación incorrecta; los metales y los organismos vivos; metales preciosos; diferencia entre vertederos: rellenos sanitarios y vertederos controlados; entre otros. Esta discusión dio lugar a algunas preguntas sobre cómo esta realidad es debatida en la sociedad. Por ejemplo, preguntado a los estudiantes cómo desechaban toda la basura electrónica, todos contestaron que la tiraban en la basura doméstica. Algunos han argumentado que no conocían otra manera de descartarla y otros dijeron que nunca habían pensado en eso.

En cuanto a las discusiones sobre la Educación Científica, específicamente relacionando informática con otras áreas del conocimiento, se hizo hincapié en que las tecnologías se han vuelto muy importantes para la formación general del alumnado. También se discutió que el profesional de la informática, así como cualquier profesional, debe tener conocimientos más allá de los conceptos técnicos y científicos de su área, destacándose una formación política, filosófica y cultural más amplia, con el fin de permitir acciones críticas y autónomas frente a los problemas de la sociedad. Durante la realización de las discusiones grupales, cuando se les preguntó a los alumnos por la elección del curso, la mayoría afirmó haber elegido el curso que más deseaban de la formación técnica básica. Aún así, no creen estar preparados para incorporarse al mercado laboral, debido a la complejidad del curso y por considerar insuficiente la duración de las disciplinas técnicas.

A través de la evaluación interdisciplinaria aplicada a los estudiantes, abordando contenidos de la educación científica y ecológica, incluyendo reflexiones éticas e históricas sobre el tema de la eliminación, manejo y 
reciclaje de desechos electrónicos, pudimos analizar y percibir que, en general, a los estudiantes les gustaba la relación de estos asuntos con su experiencia diaria así como con los temas de actualidad y de gran impacto en la sociedad. Mencionaron gran dificultad en las más diversas áreas disciplinares. Sin embargo, cuando las disciplinas incluyen cuestiones de informática o de la experiencia cotidiana, comienzan a darse cuenta y a entender cada vez más el contenido enseñado. Además, afirmaron que la carga de trabajo del curso, específicamente en las disciplinas de informática, es escasa. También señalaron que discutir temas en otras disciplinas, vinculados al área de informática, se transforma en más aprendizaje y mayor comprensión de la relación de la tecnología con la sociedad y el contenido de las ciencias.

Los alumnos manifestaron interés por otros asuntos de actualidad y de la vida cotidiana que podrían ser trabajados en las disciplinas. Otras cuestiones mencionadas fueron: los biocombustibles y el etanol, temas enfocados en la industria de producción de etanol, puesto que es una actividad económica ejercida en la región y algunos tienen conocidos y familiares trabajando en estas áreas. Teniendo en cuenta de estas discusiones, proponemos que, al abordar las disciplinas del área básica, desde la primera mitad del curso, se discutan temas relacionados a la experiencia de los estudiantes y el área de computación. Por lo tanto, a través de la elaboración de proyectos didáctico-pedagógicos específicos, tratamos de considerar las preocupaciones que ellos trajeron al mundo del trabajo y de vida en la sociedad.

\section{CONCLUSIONES}

La educación de jóvenes y adultos que ocurre en la Red Federal de Educación Profesional e Tecnológica en Brasil, cuya propuesta integra la educación propedéutica y la formación profesional, es esencial para promover mejorías en la formación del trabajador considerando su potencial capacidad de superación de la desigualdad social brasileña. Un curso en el área de informática a veces puede parecer difícil a los estudiantes que hace mucho dejaron la escuela. De este modo, enfoques didácticos diferenciados, considerando las especificidades de estos alumnos, son muy necesarios. En el proceso de construcción del proyecto descrito, los profesores en formación inicial tuvieron contacto con otras formas de planificación de clases. El enfoque de las vivencias de los alumnos disminuye la fragmentación del contenido y permite el diálogo entre diversas disciplinas, además de permitir la contextualización.

Las interacciones sociales entre los docentes con distintas formaciones contribuyen para la toma de conciencia de las medidas adoptadas con miras a mejorar la planificación de acciones futuras. El reconocimiento de las especificidades de la educación de jóvenes y adultos permite al profesor, partiendo de estudios teóricos, repensar y cambiar la práctica. Por lo tanto, los cursos de formación docente deberían contribuir para la interrelación entre la teoría y la práctica, vinculando las acciones de los profesores en la formación inicial y continuada. Consideramos, por lo tanto, que núcleos de estudios permanentes y programas como el PIBID pueden ofrecer apoyo teórico y práctico para los profesores en formación inicial y para aquellos que se encuentran en el aula, impulsándolos a salir del aislamiento de su aprendizaje hacia la acción para compartir experiencias de promoción de la construcción colectiva del conocimiento.

\section{AGRADECIMIENTOS}

Instituto Federal de Educação, Ciência e Tecnologia de Goiás (IFG), Campus Inhumas; Programa institucional de apoio à produtividade da pesquisa (ProAPP/IFG); y Coordenação de Aperfeiçoamento de Pessoal de Nível Superior (CAPES).

\section{REFERENCIAS}

Decreto n. 5.840, de 13 de julho de 2006. Institui, no âmbito federal, o Programa Nacional de Integração da Educação Profissional com a Educação Básica na Modalidade de Educação de Jovens e Adultos PROEJA, e dá outras providências. Diário Oficial [da] República Federativa do Brasil, Brasília, DF, 13 jul. 2006. Disponível em: <http://www.planalto.gov.br/ccivil_03/_ato2004-2006/2006/Decreto/D5840.htm>.

Barros, V.F.A, R.L. Costa y D.M. Castro Filho, Construção de Objeto de Aprendizagem para o Ensino da Educação Ambiental. Proceedings of International Conference on Engineering and Computer Education, 8, 389-393, Luanda, Angola (2013).

Cachapuz, A., D. Gil-Perez, A.M.P. Carvalho y A. Vilches (Orgs), A Necessária Renovação do Ensino de Ciências. São Paulo: Cortez (2005).

Costa, L.S.O. Análise da elaboração conceitual nos processos de ensino-aprendizagem em aulas de química para jovens e adultos: por uma formação integrada. 2010. 99 f. Dissertação (Mestrado em Educação em Ciências e Matemática) - Universidade Federal de Goiás, Goiânia (2010). 
Costa, L.S.O., V.F.A. Barros, R.L. Costa, L.P. Marques, T.A.S. Moraes y A.L.B Lucas, Informática e Ensino de Ciências: A problemática Ambiental do Lixo Eletroeletrônico, Memorias del Simposio Ibero-Americano de Aplicaciones y Tecnologías de Información y Comunicaciones, 1, 80-84, Orlando, Florida (2012).

Duarte, N. Conhecimento tácito e conhecimento escolar na formação do professor (por que Donald Schön não entendeu Luria). Educ. Soc., Campinas, v. 24, n. 83, pp. 601-625, ago. (2003).

Echeverría, A.R., M.C. dos Santos, L.S.O. Costa. La crisis de los alimentos y el pan: abordaje de los fenómenos químicos y sociales. Educación Química, v. 12:2012, pp. 4-11 (2012).

Freire, P. Pedagogia do oprimido. 42. ed. Rio de Janeiro: Paz e Terra (2005).

Gómez, C.M., G. Frigotto, M. Arruda, M. Arroyo y P. Nosella, Trabalho e conhecimento: dilemas na educação do trabalhador. 4. ed. São Paulo: Cortez, (2002).

IBGE. Instituto Brasileiro de Geografia e Estatística. Síntese dos indicadores sociais-uma análise das condições de vida da população brasileira, (2010).

Lima, F.B.G. de. Formação de professores nos Institutos Federais: perfil de oferta. Revista EIXO, Brasília, DF, v. 2, n. 1, p. 83-105, jan./jun (2013).

Mortimer, E.F., A.H. Machado y L.I. Romanelli, A proposta curricular de Química do Estado de Minas Gerais: Fundamentos e pressupostos. Química Nova, 23(2): 273-283 (2010).

Oliveira, A.R., E.R. Cidral, F.L. Moura, F.A. Ferreira, I.M. Riegel, L. Colossi y V.F.A. Barros, Educação Ambiental no Contexto Tecnológico: criação de um fliperama utilizando lixo eletrônico, Safety Health and Environment World Congress, 13, 290-294, Porto, Portugal (2013).

Pereira, E. M. de A. Professor como pesquisador: o enfoque da pesquisa-ação na prática docente. Geraldi, C.M.G., D. Fiorentini, E.M. de A. Pereira (Orgs.). Cartografias do trabalho docente: Professor (a)Pesquisador (a). Campinas: Mercado de Letras (1998).

Porto-Gonçalves, C. W. O desafio ambiental. 2 ed. Rio de Janeiro: Record (2011).

Saviani, D. Escola e Democracia. Campinas: Autores Associados. (2008).

Schön, D. Formar professores como profissionais reflexivos. Nóvoa, A. (Org.). Os professores e a sua formação. 3. ed. Lisboa: Dom Quixote. (1997).

Tardif, M. Saberes docentes e formação profissional. Petrópolis: Vozes. (2002).

Tripp, D. Pesquisa-ação: uma introdução metodológica. Educação e Pesquisa, São Paulo, v. 31, n. 3, p. 443-466, set./dez (2005).

Vigotski, L.S. A construção do pensamento e da linguagem. São Paulo: Martins Fontes, 496 p. (2001). 
